\title{
The Role of Gluons in Dilepton Production from the Quark-Gluon Plasma
}

\author{
Ziwei Lin and C. M. Ko \\ Cyclotron Institute and Physics Department, Texas A\&M University, College Station, \\ TX 77843
}

\begin{abstract}
We study high mass dilepton production from gluon-induced processes, $g q \rightarrow$ $q \gamma^{*}, g \bar{q} \rightarrow \bar{q} \gamma^{*}$, and $g g \rightarrow q \bar{q} \gamma^{*}$, in a thermally equilibrated but chemically under-saturated partonic matter that is expected to be created in the initial stage of ultra-relativistic heavy ion collisions. Regulating the divergence in these processes by the thermal quark mass, we find that gluon-induced processes are more important than the leading-order $q \bar{q}$ annihilation process as a result of the larger number of gluons than quarks in the partonic matter. The dependence of the thermal dilepton yield from the partonic stage of heavy ion collisions on the initial conditions for the partonic matter is also studied. We further discuss the feasibility of observing thermal dileptons from the quark-gluon plasma in heavy ion experiments.
\end{abstract}

\section{Introduction}

One of the signatures for the formation of a quark-gluon plasma (QGP) in heavy ion collisions at the Relativistic Heavy Ion Collider (RHIC) is the thermal dileptons it produces. Many studies have been carried out to estimate the dilepton yield from the QGP. In earlier works, contributions from the leading-order (LO) $q \bar{q}$ annihilation process [1] and processes up to $\mathcal{O}\left(\alpha_{s}\right)$ [2, 3] have been evaluated for a thermally and chemically equilibrated QGP. Although thermal equilibrium can be reached in the partonic matter created in heavy ion collisions at RHIC energies, chemical equilibrium is, however, unlikely to be established [4, 5]. Later studies have thus included the effects of chemical non-equilibrium on thermal dilepton and photon production from QGP [6, 7, 8, 9].

Since the partonic matter is dominated by gluons, the thermal dilepton yield from the LO $q \bar{q}$ annihilation process is thus much smaller than that from the initial DrellYan process and final charm decays [10]. However, the dominance of gluons in the initial partonic matter provides the interesting possibility that dilepton production from gluon-induced processes may become more important than the LO $q \bar{q}$ annihilation process, even though they are suppressed by powers of $\alpha_{s}$. In this paper, we shall study high mass thermal dilepton production from gluon-induced processes, i.e., the Compton processes involving one gluon in the initial state $\left(g q \rightarrow q \gamma^{*}\right.$ and $\left.g \bar{q} \rightarrow \bar{q} \gamma^{*}\right)$ and the two-gluon fusion process $\left(g g \rightarrow q \bar{q} \gamma^{*}\right)$. In particular, we shall consider these processes only at leading-order in $\alpha_{s}$, i.e., at $\mathcal{O}\left(\alpha_{s}\right)$ and $\mathcal{O}\left(\alpha_{s}^{2}\right)$ for the Compton and two-gluon fusion processes, respectively. 
This paper is organized as follows. In Section 2, we introduce the thermal quark mass in a non-equilibrium partonic matter at finite temperature, which will be used in Section 3 to regularize the divergent dilepton production cross sections for gluoninduced reactions. Thermal dilepton rates are then calculated in Section $⿴$ t for an under-saturated partonic matter in thermal equilibrium. In Section 5, we estimate the total thermal dilepton yield in heavy ion collisions at RHIC energies and study its dependence on the initial conditions for the partonic matter. We then compare it with the Drell-Yan yield to see if thermal dileptons from the QGP can be observed in experiments. Finally, a summary and discussions are given in Section 6 .

\section{The Thermal Quark Mass}

For a partonic matter in thermal equilibrium at temperature $T$ but out of chemical equilibrium, the parton distribution can be written in the Jüttner form,

$$
f_{i}=\frac{\lambda_{i}}{e^{\beta u \cdot p} \pm \lambda_{i}},
$$

where $i$ denotes different parton species, $\beta=1 / T$, and $u$ is the four velocity of the local comoving frame. In the limit of small fugacities $\left(\lambda_{i} \ll 1\right)$, the parton distribution functions reduce to the Boltzmann form,

$$
f_{i} \simeq \lambda_{i} e^{-\beta u \cdot p} .
$$

Assuming that the partonic matter is both baryon and flavor symmetric with $N_{f}$ quark flavors, i.e., $\lambda_{q_{i}}=\lambda_{\bar{q}_{i}}=\lambda_{q}\left(i=1, N_{f}\right)$, then two fugacity parameters, $\lambda_{g}$ and $\lambda_{q}$, are needed to specify the degree of chemical equilibrium.

Since chemical equilibration of the partonic matter created in heavy ion collisions at RHIC is likely to be slow, we shall use the Boltzmann distribution of Eq.(2) in the following calculations unless specified otherwise. In this case, the parton density in the local comoving frame is directly related to the fugacity,

$$
n_{g}=\lambda_{g} \frac{d_{g} T^{3}}{\pi^{2}}, \quad n_{q}+n_{\bar{q}}=\lambda_{q} \frac{\left(d_{q}+d_{\bar{q}}\right) N_{f} T^{3}}{\pi^{2}},
$$

if partons are taken to be massless. In the above, $d_{i}$ is the degeneracy factor of parton $i$, i.e., $d_{g}=2\left(N_{c}^{2}-1\right)$ and $d_{q}=d_{\bar{q}}=2 N_{c}$.

The thermal quark mass, denoted as $\mu$, is given by

$$
\mu^{2}=4 \pi \alpha_{s} \frac{N_{c}^{2}-1}{2 N_{c}} \int \frac{d^{3} p}{(2 \pi)^{3}} \frac{1}{p}\left(f_{g}+f_{q}\right),
$$

where $\alpha_{s}=g^{2} /(4 \pi)$ is the QCD strong coupling. For a QGP in chemical equilibrium, this gives a thermal quark mass $\mu^{2}=g^{2} T^{2} / 6$ when using the exact parton distribution of Eq.(11). For an under-saturated partonic matter described approximately by Eq.(2), the thermal quark mass becomes

$$
\mu^{2}=\frac{2 g^{2} T^{2}}{3 \pi^{2}}\left(\lambda_{g}+\lambda_{q}\right) .
$$




\section{Dilepton Production Cross Sections}

Leading-order processes in the strong coupling constant $\alpha_{s}$ for dilepton production from parton-parton interactions are $q \bar{q}$ annihilation $\left(q \bar{q} \rightarrow \gamma^{*}\right)$, Compton processes $(g q \rightarrow$ $q \gamma^{*}$ and $\left.g \bar{q} \rightarrow \bar{q} \gamma^{*}\right)$, two-gluon fusion $\left(g g \rightarrow q \bar{q} \gamma^{*}\right)$, and two-quark $\left(q q \rightarrow q q \gamma^{*}\right)$ and twoantiquark bremsstrahlung $\left(\bar{q} \bar{q} \rightarrow \bar{q} \bar{q} \gamma^{*}\right)$. They are of order $\mathcal{O}(1), \mathcal{O}\left(\alpha_{s}\right), \mathcal{O}\left(\alpha_{s}^{2}\right), \mathcal{O}\left(\alpha_{s}^{2}\right)$ and $\mathcal{O}\left(\alpha_{s}^{2}\right)$, respectively. In the present study, we neglect two-quark and two-antiquark bremsstrahlung as they do not involve gluons in the initial state and are also suppressed by $\alpha_{s}^{2}$ relative to the LO $q \bar{q}$ annihilation process. Furthermore, we neglect $3 \rightarrow 2$ processes as they are expected to be less important when parton fugacities are much below one. The processes included in our study for dilepton production are shown in Fig.11.

The cross section for dilepton production from partons can be generally written as

$$
\frac{d \hat{\sigma}_{i j}}{d M^{2}}=\frac{4 \pi \alpha^{2}}{9 M^{2} \hat{s}} W_{i j}
$$

In the above, indices $i$ and $j$ specify initial-state partons, $\hat{s}=\left(p_{i}+p_{j}\right)^{2}$, and $\alpha$ is the fine structure constant. The quantity $W_{i j}$ is related to the square of the transition amplitude. For the leading-order $q \bar{q}$ annihilation process, it is simply

$$
W_{q \bar{q}}=e_{q}^{2} \delta(1-\xi)
$$

with $\xi=M^{2} / \hat{s}$.

For the Compton processes, the squared matrix element, after averaging over initial and summing over final spins and colors, is given by

$$
\overline{\left|M_{g q \rightarrow q \gamma^{*}}\right|^{2}}=\overline{\left|M_{g \bar{q} \rightarrow \bar{q} \gamma^{*}}\right|^{2}}=\frac{(4 \pi)^{2} \alpha \alpha_{s} e_{q}^{2}}{3}\left[-\frac{\hat{t}}{\hat{s}}-\frac{\hat{s}}{\hat{t}}+\frac{2 M^{2}\left(\hat{s}+\hat{t}-M^{2}\right)}{\hat{s} \hat{t}}\right] .
$$

The total cross section, given by $\hat{\sigma}=\int d \hat{t} \overline{|M|^{2}} /\left(16 \pi \hat{s}^{2}\right)$, is divergent. Following previous studies of low mass dilepton and photon production from the QGP [11, 12, 13], we use the thermal quark mass as the screening mass for regularizing all divergence in the cross sections. We thus replace $1 / \hat{t}$ with $1 /\left(\hat{t}-\mu^{2}\right)$, and Eq. (8) then becomes

$$
\overline{\left|M_{g q \rightarrow q \gamma^{*}}\right|^{2}} \simeq \frac{(4 \pi)^{2} \alpha \alpha_{s} e_{q}^{2}}{3}\left[-\frac{\hat{t}}{\hat{s}}-\frac{\hat{s}}{\hat{t}-\mu^{2}}+\frac{2 M^{2}\left(\hat{s}+\hat{t}-M^{2}\right)}{\hat{s}\left(\hat{t}-\mu^{2}\right)}\right] .
$$

After integrating over $\hat{t}$, we obtain

$$
W_{g q}=W_{g \bar{q}}=\frac{\alpha_{s} e_{q}^{2}}{4 \pi} c_{0}(\xi)
$$

where

$$
c_{0}(\xi)=\frac{1+2 \xi-3 \xi^{2}}{2}+\left[1-2 \xi\left(1-\xi+\frac{\mu^{2}}{M^{2}} \xi\right)\right] \ln \left(1+\frac{M^{2}}{\mu^{2}} \frac{1-\xi}{\xi}\right) .
$$


There are no studies on dilepton production from the two-gluon fusion process, $g g \rightarrow q \bar{q} \gamma^{*}$, at finite temperature. However, processes involving heavy quarks in the final state such as $g g \rightarrow Q \bar{Q} \gamma^{*}$ have been studied in determining the K-factor in DrellYan processes in the limit of $M^{2} \gg m_{Q}^{2}$ [14, 15]. These results can be adopted for the production of dileptons with invariant mass $M \geq 2 \mathrm{GeV}$. Since $M^{2} \gg \mu^{2}$ unless the temperature is extremely high, we can replace the heavy quark mass $m_{Q}$ in Ref. [14, 15] with the thermal quark mass $\mu$ and obtain

$$
W_{g g}=e_{q}^{2}\left(\frac{1}{2}\right)^{2}\left(\frac{\alpha_{s}}{4 \pi}\right)^{2}\left[c_{1}(\xi) \ln ^{2} \frac{M^{2}}{\mu^{2}}+c_{2}(\xi) \ln \frac{M^{2}}{\mu^{2}}+c_{3}(\xi)\right]
$$

In the above, the functions $c_{1}(\xi), c_{2}(\xi)$ and $c_{3}(\xi)$ can be obtained from Ref. [14], where the calculation was carried out in the $\overline{\mathrm{MS}}$ scheme.

\section{Thermal Dilepton Rates}

For a partonic matter in thermal equilibrium at temperature $T$ but out of chemical equilibrium, the thermal dilepton rate is given by

$$
\frac{d R_{i j}}{d M}=S_{i j} \sum \int \frac{d^{3} p_{1}}{(2 \pi)^{3}} f_{i}\left(p_{1}\right) \frac{d^{3} p_{2}}{(2 \pi)^{3}} f_{j}\left(p_{2}\right) v_{\mathrm{rel}} \frac{d \hat{\sigma}_{i j}}{d M}(M, \sqrt{\hat{s}})
$$

with $v_{\text {rel }}=\left(p_{1} \cdot p_{2}\right) /\left(p_{1} p_{2}\right), S_{g g}=1 / 2$ accounting for the two identical gluons in the initial state, and $S_{i j}=1$ otherwise. The summation is over the parton spin and color indices.

The integrals in the above equation can be evaluated using the rapidity and transverse momentum variables,

$$
\begin{aligned}
& \hat{s}=M_{0}^{2}, \quad P_{0}^{\mu}=\left(M_{\perp 0} \cosh Y_{0}, P_{\perp 0} \cos \Phi_{0}, P_{\perp 0} \sin \Phi_{0}, M_{\perp 0} \sinh Y_{0}\right) \\
& p_{1}^{\mu}=p_{\perp 1}\left(\cosh y_{1}, \cos \phi_{1}, \sin \phi_{1}, \sinh y_{1}\right)
\end{aligned}
$$

where $M_{\perp 0}=\sqrt{M_{0}^{2}+P_{\perp 0}^{2}}$. Inserting $\int d^{4} P_{0} \delta^{4}\left(P_{0}-p_{1}-p_{2}\right)$ into Eq.(13), the delta function can then be used to remove the $\int d^{3} p_{2}$ and $\int d p_{\perp 1}$ integrals. After integrating over $\Phi_{0}$ and redefining $y_{1}-Y_{0}$ and $\phi_{1}-\Phi_{0}$ as $y_{1}$ and $\phi_{1}$, we obtain

$$
\begin{aligned}
\frac{d R_{i j}}{d M}= & \frac{d_{i} d_{j} \lambda_{i} \lambda_{j} S_{i j}}{4(2 \pi)^{5}} \int d M_{0} d Y_{0} d M_{\perp 0} d \phi_{1} d y_{1} \\
& \times \frac{M_{\perp 0} M_{0}^{5} e^{-M_{\perp 0} \cosh \left(Y_{0}-\eta\right) / T}}{\left(M_{\perp 0} \cosh y_{1}-P_{\perp 0} \cos \phi_{1}\right)^{2}} \frac{d \hat{\sigma}_{i j}}{d M}\left(M, M_{0}\right)
\end{aligned}
$$

Carrying out the integrals over $Y_{0}$ and $\phi_{1}$ and then those over $y_{1}$ and $M_{\perp 0}$ gives

$$
\frac{d R_{i j}}{d M}=\frac{d_{i} d_{j} \lambda_{i} \lambda_{j} S_{i j}}{(2 \pi)^{4}} \int_{M} d M_{0} M_{0}^{4} T K_{1}\left(M_{0} / T\right) \frac{d \hat{\sigma}_{i j}}{d M}\left(M, M_{0}\right) .
$$


Summing over quark flavors, we finally have

$$
\begin{aligned}
\frac{d R_{q \bar{q}}}{d M}= & \alpha^{2} \lambda_{q}^{2} \frac{F_{q}}{\pi^{3}} M^{2} T K_{1}(M / T), \\
\frac{d R_{g q+g \bar{q}}}{d M}= & \alpha^{2} \alpha_{s} \lambda_{g} \lambda_{q} \frac{8 F_{q}}{3 \pi^{4}} \frac{T}{M} \int_{M} d M_{0} M_{0}^{2} K_{1}\left(M_{0} / T\right) c_{0}(\xi), \\
\frac{d R_{g g}}{d M}= & \alpha^{2} \alpha_{s}^{2} \lambda_{g}^{2} \frac{F_{q}}{9 \pi^{5}} \frac{T}{M} \int_{M} d M_{0} M_{0}^{2} K_{1}\left(M_{0} / T\right) \\
& \times\left[c_{1}(\xi) \ln ^{2} \frac{M^{2}}{\mu^{2}}+c_{2}(\xi) \ln \frac{M^{2}}{\mu^{2}}+c_{3}(\xi)\right],
\end{aligned}
$$

with

$$
F_{q}=\sum_{i=1}^{N_{f}} e_{q_{i}}^{2}=\frac{5}{9} \text { for } N_{f}=2 .
$$

For the strong coupling constant, we use the LO value with three quark flavors,

$$
\alpha_{s}=\frac{4 \pi}{9 \ln \frac{M^{2}}{\Lambda^{2}}}
$$

with $\Lambda=200 \mathrm{MeV}$ and the normalization scale chosen to be the dilepton mass $M$.

In the limit $M^{2} \gg \mu^{2}$, the thermal dilepton rates depend on the coupling constant and fugacities as

$$
\begin{aligned}
\frac{d R_{q \bar{q}}}{d M} & \propto \alpha^{2} \lambda_{q}^{2} \\
\frac{d R_{g q+g \bar{q}}}{d M} & \propto \alpha^{2} \alpha_{s} \lambda_{g} \lambda_{q} \ln \frac{1}{\lambda_{g}+\lambda_{q}} \\
\frac{d R_{g g}}{d M} & \propto \alpha^{2} \alpha_{s}^{2} \lambda_{g}^{2} \ln ^{2} \frac{1}{\lambda_{g}+\lambda_{q}}
\end{aligned}
$$

In Fig. 2, we show the thermal dilepton rates from a quark-gluon plasma in full thermal and chemical equilibrium at $T=570 \mathrm{MeV}$. It is seen that for dileptons with mass above $2 \mathrm{GeV}$ the $\mathrm{LO} q \bar{q}$ annihilation process gives the dominant contribution, while the Compton processes give a small contribution, and that from two-gluon fusion process is even smaller. These results are not unexpected as the Compton and twogluon fusion processes are suppressed by $\alpha_{s}$ and $\alpha_{s}^{2}$, respectively.

The situation is, however, very different if partons are not in chemical equilibrium. As an example, we show in Fig. 3 the thermal dilepton rates for $T=570 \mathrm{MeV}$, $\lambda_{g}=0.060$ and $\lambda_{q}=0.0072$. The reason for choosing these values will be given in the next Section. In this case, the Compton processes produce more thermal dileptons than the LO $q \bar{q}$ annihilation, and even two-gluon fusion processes give a comparable contribution to lower-mass dileptons. As a result, the total thermal dilepton yield is significantly enhanced by gluonic processes.

The appreciable difference between the results shown in Fig. 3 and Fig. 2 can be qualitatively understood from the scaling behavior given in Eq.(22). Because of 
the quadratic dependence on parton fugacities, gluonic processes become dominant over the $q \bar{q}$ process when gluons greatly outnumber quarks $\left(\lambda_{g} / \lambda_{q} \gg 1\right)$. Even if $\lambda_{g} / \lambda_{q}$ is fixed but $\lambda_{g} \rightarrow 0$ in an under-saturated partonic matter, both $R_{g q+g \bar{q}} / R_{q \bar{q}}$ and $R_{g g} / R_{q \bar{q}}$ become logarithmic divergent, indicating that gluonic processes become more important than $q \bar{q}$ annihilation when the parton system is further away from chemical equilibrium.

To illustrate this quantitatively, we show in Fig. 1 the full-equilibrium results in Fig. 2 multiplied by the quadratic fugacity factors. In this case, the Compton processes are already as important as the $q \bar{q}$ annihilation process. Further increase of the gluonic contribution when going from Fig. H to Fig. 3 is due to the logarithmic terms in Eq.(22), which come from the thermal quark mass in the regularized partonic cross sections given by Eqs.(10-12).

We also see in Figs. 2-4 that the LO $q \bar{q}$ annihilation process is most effective in producing high invariant-mass dileptons because all incoming energy can be used. In contrast, there are one or two partons in the final state in the Compton and twogluon fusion processes to carry away part of the incoming energy. To produce high mass dileptons from these processes thus requires more energetic partons, which are less available. Therefore, the three curves in Fig. 3 corresponding to the above three processes exhibit increasingly larger slopes.

\section{Thermal Dilepton Yield at RHIC}

In this Section, we estimate the thermal dilepton yield in central $\mathrm{Au}+\mathrm{Au}$ collisions at RHIC by integrating the thermal dilepton rate over the space-time four volume, which will be taken from a schematic model. Also, we will study the feasibility of observing the thermal dilepton signal in experiments by comparing it with the Drell-Yan background.

Starting from Eq.(15) and using $d^{4} x=\tau d \tau d \eta d^{2} x_{\perp}$, we first integrate over $\eta$ instead of $Y_{0}$ and then over $\phi_{1}, y_{1}$ and $M_{\perp 0}$ as in previous section. This gives

$$
\frac{d N_{i j}}{d M}=\int d Y_{0} d \tau \tau d^{2} x_{\perp} \frac{d_{i} d_{j} \lambda_{i} \lambda_{j} S_{i j}}{(2 \pi)^{4}} \int_{M} d M_{0} M_{0}^{4} T K_{1}\left(M_{0} / T\right) \frac{d \hat{\sigma}_{i j}}{d M}\left(M, M_{0}\right),
$$

which then leads to

$$
\frac{d N_{i j}}{d M^{2} d Y_{0}}=\int_{\tau_{0}}^{\tau_{f}} d \tau \tau d^{2} x_{\perp} \frac{d R_{i j}}{d M^{2}}(\tau)
$$

In the above, $\tau_{0}$ is the initial proper time when the parton system first reaches thermal equilibrium, and $\tau_{f}$ is the final proper time when the temperature drops to $T_{c}$ (chosen to be $200 \mathrm{MeV}$ ) and the partonic matter makes a transition to the hadronic matter. The rate $d R_{i j} / d M^{2}$ depends on $\tau$ implicitly through the time evolution of the variables $\lambda_{i}, T$ and $\mu$.

We note that while the pair rapidity $Y_{0}$ of the two partons in the initial state is equal to the pair rapidity $Y$ of the dilepton in the LO $q \bar{q}$ annihilation process, they are different in gluon-induced processes. However, if we assume that the expansion of the 
partonic matter follows the Bjorken scaling, the dependence of the thermal dilepton yield on $Y$ and $Y_{0}$ must have the following functional form:

$$
\frac{d N}{d M^{2} d Y d Y_{0}}=F\left(Y-Y_{0}, M\right)
$$

as a result of boost invariance of the integrand on the right-hand side of Eq.(13). This gives

$$
\frac{d N}{d M^{2} d Y}=\frac{d N}{d M^{2} d Y_{0}}
$$

The same argument applies to $\eta$, and it is easy to verify that

$$
\frac{d N}{d M^{2} d \eta}=\frac{d N}{d M^{2} d Y_{0}}
$$

\subsection{Time evolution of parton fugacities and temperature}

First, we need to specify the time evolution of partons in the quark-gluon phase created in heavy ion collisions at RHIC. Earlier studies [10] based on kinetic equations including $2 \leftrightarrow 3$ gluonic processes have shown that thermal equilibrium is achieved first, and both gluon and quark fugacities increase as the system evolves. However, since the quarkgluon plasma has only a finite lifetime of a few $\mathrm{fm} / c$, chemical equilibrium is unlikely to be established, and parton fugacities thus remain well below the value of one. To take into account such effects, we follow Fig. 25 of Ref.[10] by simply assuming that the parton fugacities increase linearly with the proper time,

$$
\lambda_{i}=\lambda_{i, 0}\left(\frac{\tau}{\tau_{0}}\right) .
$$

In an ideal hydrodynamic description, temperature of the partonic matter decreases as $T \propto 1 / \tau^{1 / 3}$, which then leads to a decrease of the rapidity density of transverse energy as $d E_{\mathrm{T}} / d y \propto \tau T^{4} \propto 1 / \tau^{1 / 3}$. In the parton cascade model, the time evolution of the rapidity density of transverse energy depends sensitively on the magnitude of parton cross sections and densities [16]. Since we do not expect the rapidity density of transverse energy to change more than $25 \%$ during the partonic stage of RHIC heavy ion collisions [16, 17], we assume for simplicity that $d E_{\mathrm{T}} / d y$, given by

$$
\frac{d E_{\mathrm{T}}}{d y}=\frac{d N}{d y}<p_{\perp}>\propto\left(\sum_{i} d_{i} \lambda_{i, 0}\right) \tau T^{3} T \propto\left(\tau T^{2}\right)^{2},
$$

is a constant. This then leads to the time evolution of temperature as

$$
T=T_{0}\left(\frac{\tau_{0}}{\tau}\right)^{1 / 2} .
$$

In the above, we have used

$$
\frac{d N}{d y}=\tau_{0} \pi R_{A}^{2} \sum_{i} n_{i}
$$


at central rapidity at $\tau_{0}$ and $R_{A} \simeq 1.12 A^{1 / 3} \mathrm{fm}$. Also, we neglect the transverse expansion throughout this study. The effect of transverse expansion on the production of dileptons of mass above $2 \mathrm{GeV}$ has been shown to be very small in heavy ion collisions at RHIC energies [18]. This is expected because high mass dileptons are mainly produced in the early stage of the partonic matter, when the transverse flow has not much developed.

In Fig. 5 the time evolution of fugacities and temperature given in Eqs.(28) and (30) are shown. We see that the temperature decreases faster than that given by the scenario of entropy conservation, i.e., $T \propto 1 / \tau^{1 / 3}$, as parton production increases the entropy.

\subsection{The default scenario}

As in Ref. [10], where the parton equilibration is studied using kinetic equations, we assume that at the proper time $\tau_{0}=0.7 \mathrm{fm} / c$, thermal equilibrium is established at a temperature $T_{0}=570 \mathrm{MeV}$. We also take the same gluon and quark densities at $\tau_{0}$ as in Ref. [10], and obtain the following values for the initial parton fugacities [19]:

$$
\lambda_{g, 0}=0.060 \text { and } \lambda_{q, 0}=0.0072 \text {. }
$$

With these values, we find that quarks and antiquarks make about $15 \%$ of the partons and the thermal quark mass is about $75 \mathrm{MeV}$ if the strong coupling constant is evaluated at the normalization scale of $2 \mathrm{GeV}$. Subsequent expansion of the partonic matter is assumed to follow the Bjorken scaling.

We have evaluated the thermal dilepton yield using Eq.(24), and the results are shown in Fig. 6. Also shown is the yield from primary Drell-Yan hard collisions using a K-factor of 1.7, obtained from fitting the E772 data, to take into account higherorder contributions. As in thermal dilepton rates shown in Fig. 3, we find that the Compton processes produce more dileptons than both the LO $q \bar{q}$ annihilation and the two-gluon fusion processes. This also indicates that the conventional way of using a $\mathrm{K}$ factor of 2 to account for all higher-order contributions [8] underestimates the possible large enhancement from gluonic processes. We note that, despite the enhancement from gluonic processes, the total thermal dilepton yield is still a factor of 30 or more (depending on the dilepton mass) below the Drell-Yan yield. It is thus unlikely that thermal dileptons from the partonic matter can be observed in heavy ion collisions at RHIC.

\subsection{An upper limit}

The small thermal dilepton yield discussed in the above default scenario is mainly due to the small number of quarks and antiquarks compared to that of gluons assumed in the initial conditions for the partonic matter. While we have used the ratio of initial quarks to gluons that is consistent with minijet calculations based on perturbative QCD together with initial and final state radiations, it is possible that additional soft

partons may be produced. Also, there may be already some chemical equilibration 
before the thermal stage starts at $\tau_{0}$. Both could then lead to initial quark fugacity that is larger than the estimate from minijet calculations.

To obtain an upper limit for the thermal dilepton yield, we assume that quarks and antiquarks are in relative chemical equilibrium with gluons at the initial time $\tau_{0}$, i.e., $\lambda_{q}=\lambda_{g}$, although both are still out of full chemical equilibrium. Requiring the same value of total $d E_{\mathrm{T}} / d y$ at central rapidity as before and using the same $\tau_{0}$ and $T_{0}$, we obtain from Eq.(29) the following initial parton fugacities for the upper limit (UL):

$$
\lambda_{g, 0}^{U L}=\lambda_{q, 0}^{U L}=\frac{d_{g} \lambda_{g, 0}+\left(d_{q}+d_{\bar{q}}\right) N_{f} \lambda_{q, 0}}{d_{g}+\left(d_{q}+d_{\bar{q}}\right) N_{f}} \simeq 0.028
$$

This gives a ratio of $3 / 5$ for the sum of quarks and antiquarks to the total number of partons, similar to that in an ideal gas of gluons and quarks with $N_{f}=2$.

Using the same time evolution as given by Eqs.(28) and (30), we have evaluated the thermal dilepton yield with the above initial parton conditions, and the results are shown in Fig.7. Because of increased number of quarks and antiquarks, the contribution from the LO $q \bar{q}$ process is increased by a factor of about 16, while gluonic processes become less important in comparison. As a result, the total thermal dilepton yield is about a factor of six higher than that in the default scenario. Depending on the dilepton mass, this is still about a factor of 5 or more below the Drell-Yan yield.

\subsection{Dependence on initial parton conditions}

The thermal dilepton yield is sensitive to the initial conditions, such as $\tau_{0}$ and $T_{0}$, for the partonic matter. For example, the contribution from $q \bar{q}$ annihilation given in Eq.(17) depends on temperature as

$$
\frac{d R_{q \bar{q}}}{d M} \propto T^{3 / 2} e^{-M / T}
$$

The thermal dilepton yield can be large if the partonic matter starts at a very high initial temperature $T_{0}$. In the following, we study the dependence of the thermal dilepton yield on the parameters $\lambda_{g, 0}, \lambda_{q, 0}, \tau_{0}$ and $T_{0}$.

For a fully-equilibrated QGP at $T_{0}=570 \mathrm{MeV}$ with $N_{f}=2$ and an initial proper time $\tau_{0}=0.7 \mathrm{fm} / c$, the rapidity density of transverse energy is $\mathrm{U}$

$$
\frac{d E_{\mathrm{T}}}{d y}=\tau_{0} \pi R_{A}^{2} \frac{\pi}{4}\left(\frac{8}{15}+\frac{7 N_{f}}{20}\right) \pi^{2} T_{0}^{4} \sim 12,000 \mathrm{GeV}
$$

at central rapidity. This is more than an order of magnitude larger than most model estimates of the final $d E_{\mathrm{T}} / d y$.

On the other hand, Eq.(31) with $\lambda_{g, 0}=0.060, \lambda_{q, 0}=0.0072, \tau_{0}=0.7 \mathrm{fm} / c$ and $T_{0}=570 \mathrm{MeV}$ in the default scenario gives at central rapidity

$$
\frac{d E_{\mathrm{T}}}{d y}=\frac{d N}{d y} \frac{3 \pi T}{4} \sim 350 \mathrm{GeV}
$$

\footnotetext{
${ }^{1}$ Bose-Einstein and Fermi-Dirac distributions are used.
} 
Including additional $E_{\mathrm{T}}$ from subsequent string fragmentation gives a final $d E_{\mathrm{T}}^{F} / d y \sim$ $900 \mathrm{GeV}$ at central rapidity, which is comparable with predictions from most event generators and cascade models for heavy ion collisions at RHIC energies.

In the following, we shall study the dependence of thermal dilepton yields on the initial conditions that have same value of initial $d E_{\mathrm{T}} / d y$ at central rapidity. According to Eq.(29), this is equivalent to taking initial conditions with the constraint

$$
\left(\sum_{i} d_{i} \lambda_{i, 0}\right) \tau_{0} T_{0}^{4}=\text { const. }
$$

We have already studied the effect due to the increase of the quark fraction in the partonic matter. Here, we study the dependence on initial conditions with a fixed $\lambda_{g, 0} / \lambda_{q, 0}$, i.e., with same values of both $\lambda_{g, 0} \tau_{0} T_{0}^{4}$ and $\lambda_{q, 0} \tau_{0} T_{0}^{4}$.

Using the same time evolution given by Eqs.(28) and (30), the LO $q \bar{q}$ annihilation process gives

$$
\begin{aligned}
\frac{d N_{q \bar{q}}}{d M^{2} d Y} & \simeq \frac{\pi R_{A}^{2}}{2 M} \int_{\tau_{0}}^{\tau_{f}} d \tau \tau \frac{d R_{q \bar{q}}}{d M}(\tau) \propto \int_{\tau_{0}}^{\tau_{f}} d \tau \tau \lambda_{q}^{2} T K_{1}\left(\frac{M}{T}\right) \\
& \propto\left(\lambda_{q, 0} \tau_{0} T_{0}^{4}\right)^{2} \int_{\frac{M}{T_{0}}}^{\frac{M}{T_{c}}} d x x^{6} K_{1}(x) \propto \int_{\frac{M}{T_{0}}}^{\infty} d x x^{6} K_{1}(x)
\end{aligned}
$$

It is seen that this contribution depends only on one dimensionless variable $M / T_{0}$.

In Fig.8, we show the value of the last integral in the above equation as a function of $M / T_{0}$. As $T_{0}$ increases, the dilepton yield at a fixed mass $M$ increases as the variable $M / T_{0}$ shifts to the left, and the yield saturates at the high temperature limit. The slow increase and final saturation with increasing initial temperature $T_{0}$ deviate from the naive expectation based on Eq.(34). This is due to the constraint on the total $d E_{\mathrm{T}} / d y$ in Eq.(37), which requires that as the initial temperature increases, the initial volume and/or the initial parton fugacities become smaller, thus making the total dilepton yield less sensitive to the initial conditions. We also see that the yield of higher-mass dileptons depend more strongly on the initial temperature. However, in mass range of $2-4 \mathrm{GeV}$, where the yield of thermal dileptons is closer to the Drell-Yan yield, the variable $M / T_{0}$ has a value between 3.5 to 7 when the default choice of $T_{0}=570 \mathrm{MeV}$ is used. Fig.8 thus shows that the maximum increase one can obtain by increasing $T_{0}$ while keeping the same total $d E_{\mathrm{T}} / d y$ is only $15 \%$ at dilepton mass of $2 \mathrm{GeV}$ and no more than a factor of 2 at dilepton mass of $4 \mathrm{GeV}$. Therefore, thermal dileptons are still well below the Drell-Yan yield even if we allow the maximum increase of the upper limit results in Fig. 7 .

Same studies can be carried out for the Compton and two-gluon processes, but they involve more parameters besides the dimensionless variable $M / T_{0}$ due to the presence of the thermal quark mass $\mu$. Relations similar to Eq.(38) can still be obtained except for the logarithmic factors shown in Eq.(22). Similar conclusions thus hold as long as the screening mass is not extremely small. 


\section{Discussions and Summary}

In the present study, only leading-order processes have been considered. For lowmass dilepton production, higher-order effects have been studied [20] using the HardThermal-Loop (HTL) resummation method with effective propagators and vertices. It has been found that two-loop diagrams can give contributions at the same order as one-loop diagrams. Numerical calculations have shown that in heavy ion collisions at RHIC and LHC energies the two-loop contribution from the bremsstrahlung processes is important only for dileptons with mass $M \ll T$ and becomes less important than the leading-order contribution for dileptons with mass $M>0.3 \mathrm{GeV}$ 21]. Since our study mainly concerns dileptons with mass above $2 \mathrm{GeV}(M \gg T)$, we do not expect that the inclusion of these processes will significantly change our results.

However, there are still uncertainties in our results. First of all, the divergence in the dilepton production cross sections has been simply regularized by the thermal quark mass. Studies that take into account higher-order effects using the thermal field theory have indeed shown that for low mass dilepton or photon production in the QGP the thermal quark mass provides a self-consistent screening effect [11, 12, 13]. However, the dependence of the dilepton production cross section on the thermal quark mass differs from that based on the lowest-order perturbative calculations as used in our study. Also, using different parton distributions can lead to different dependence of the cross sections on the thermal quark mass [13]. Since we have not carried out a self-consistent calculation including higher-order effects and have used the Boltzmann distribution instead of the quantum distribution, the coefficients that appear in association with the thermal quark mass as given in Eqs.(11) and (12) are expected to be different when a more complete calculation is carried out. To illustrate this uncertainty, we show in Fig.9 the results from changing the screening mass by a factor of two but with the same default initial parton conditions. Since dilepton yields from the Compton and two-gluon processes roughly scale as $\log \mu$ and $\log ^{2} \mu$, respectively, sizable differences are seen.

We have only considered dilepton production from the stage where partons have reached thermal equilibrium. The contribution from the pre-thermal stage has not been included. Also, we have not considered chemical equilibration during the prethermal stage, which could change the initial conditions of the partonic matter such as the fugacities $\lambda_{g, 0}$ and $\lambda_{q, 0}$. With the dilepton production cross sections given in Section 3, the pre-thermal dilepton yield can be calculated using a pre-thermal parton distribution that is either parameterized or from a parton cascade code. With the latter, the entire time evolution of parton thermal and chemical equilibration can be studied, so the separation of pre-thermal and thermal stages, which is rather crude, is unnecessary. In this way, the evolution of parton fugacities can be self-consistently determined, and all secondary dileptons from the partonic stage can be calculated. In a previous study [22], we have carried out a calculation of secondary dileptons from the LO $q \bar{q}$ annihilation process within the parton cascade code ZPC [23], where initial

parton fugacities were determined directly from the HIJING model [24]. Since the default HIJING model gives too few quarks and antiquarks, with the ratio of the sum 
of quarks and antiquarks to the total number of partons being only $4.5 \%$ (compared with the ratio of $15 \%$ in the default scenario of the present study based on minijet calculations using the perturbative QCD), the thermal dilepton yield in Ref. 22 is lower than the default result in this study by a factor of 10 .

In summary, we have studied thermal dilepton production in heavy ion collisions at RHIC energies. In particular, contributions from gluonic processes and their dependence on the initial parton conditions are investigated. We find that gluonic processes significantly enhance the thermal dilepton yield as one expects more gluons than quarks in the partonic matter formed in heavy ion collisions at RHIC. However, we have also shown that with the constraint of Eq.(36) on the total initial $d E_{\mathrm{T}} / d y$ of the partonic matter, thermal dileptons are always significantly lower than those from the Drell-Yan process for any reasonable variations of the initial temperature, proper time, and fugacities. Our results show that thermal dileptons can compete with those from the Drell-Yan process only in a rather ideal case, when the initial energy density is higher than most current expectations, and parton chemical equilibration proceeds fast so that quarks are close to relative equilibrium with gluons.

Acknowledgments: We thank B. Kämpfer, W.L. van Neerven and S.M.H. Wong for helpful discussions. This work was supported in part by the National Science Foundation under Grant No. PHY-9870038, the Welch Foundation under Grant No. A-1358, and the Texas Advanced Research Project FY97-010366-068. 


\section{References}

[1] K. Kajantie, J. Kapusta, L. McLerran, A. Mekjian, Phys. Rev. D 34 (1986) 2746.

[2] R. Baier, B. Pire, D. Schiff, Phys. Rev. D 38 (1988) 2814.

[3] J. Cleymans, I. Dadić, Phys. Rev. D 47 (1993) 160.

[4] E. Shuryak, Phys. Rev. Lett. 68 (1992) 3270.

[5] T.S. Biró, E. van Doorn, B. Müller, M.H. Thoma, X.N. Wang, Phys. Rev. C 48 (1993) 1275.

[6] E. Shuryak and L. Xiong, Phys. Rev. Lett. 70 (1993) 2241.

[7] B. Kämpfer and O.P. Pavlenko, Z. Phys. C 62 (1994) 491.

[8] M. Strickland, Phys. Lett. B 331 (1994) 245.

[9] C.T. Traxler and M.H. Thoma, Phys. Rev. C 53 (1996) 1348.

[10] X.-N. Wang, Phys. Rep. 280 (1997) 287.

[11] T. Altherr and P.V. Ruuskanen, Nucl. Phys. B 380 (1992) 377.

[12] M.H. Thoma and C.T. Traxler, Phys. Rev. D 56 (1997) 198.

[13] R. Baier, M. Dirks, K. Redlich, D. Schiff, Phys. Rev. D 56 (1997) 2548.

[14] P.J. Rijken, W.L. van Neerven, Phys. Rev. D 52 (1995) 149.

[15] R. Hamberg, W.L. van Neerven, T. Matsuura, Nucl. Phys. B 359 (1991) 343; W.L. van Neerven, E.B. Zijlstra, Nucl. Phys. B 382 (1992) 11.

[16] M. Gyulassy, Y. Pang, B. Zhang, Nucl. Phys. A 626 (1997) 999.

[17] B. Zhang, C.M. Ko, B.-A. Li, Z. Lin, preprint nucl-th/9907017.

[18] D.K. Srivastava, M.G. Mustafa and B. Müller, Phys. Rev. C 56 (1997) 1064.

[19] The difference between the values of our parton fugacities $\left(\lambda_{g, 0}=0.060, \lambda_{q, 0}=\right.$ $0.0072)$ and those in Ref. [10] $\left(\lambda_{g}^{0}=0.05, \lambda_{q}^{0}=0.008\right)$ for the same parton number densities is due to the fact that we use the factorized Boltzmann distribution instead the factorized Bose-Einstein and Fermi-Dirac distributions.

[20] P. Aurenche, F. Gelis, R. Kobes and H. Zaraket, Phys. Rev. D 58 (1998) 085003; ibid 60 (1999) 076002.

[21] D. Pal and M.G. Mustafa, Phys. Rev. C 60 (1999) 034905.

[22] S. Bass et al, preprint hucl-th/9907090. 
[23] B. Zhang, Comp. Phys. Comm. 109 (1998) 193.

[24] X.-N. Wang and M. Gyulassy, Phys. Rev. D 44 (1991) 3501; ibid 45 (1992) 844. 
a)

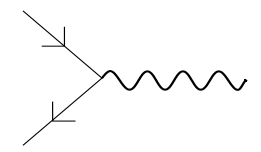

b)
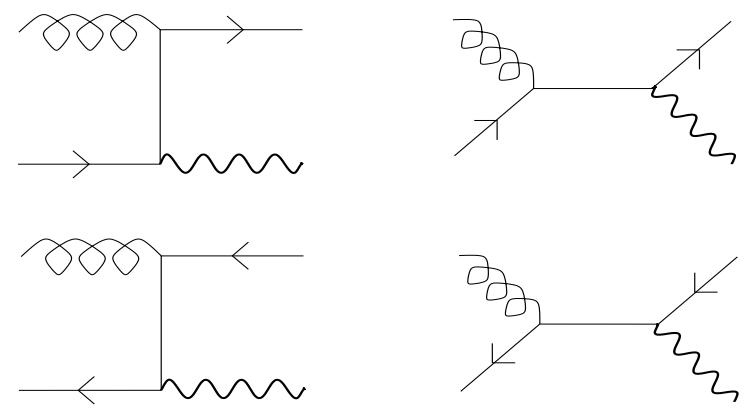

c)
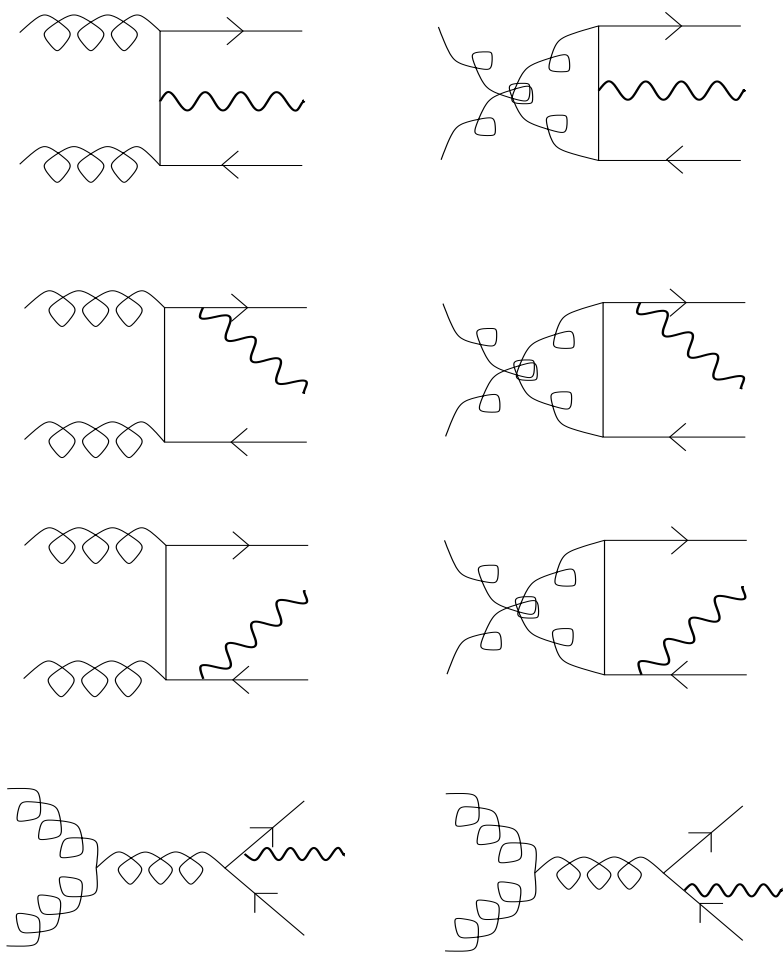

Figure 1: Diagrams for dilepton production: a) $q \bar{q}$ annihilation, b) the Compton processes, and c) two-gluon fusion processes. Curly lines denote gluons, wavy lines denote virtual photons, and straight lines with arrows to the right (left) denote quarks (antiquarks). 


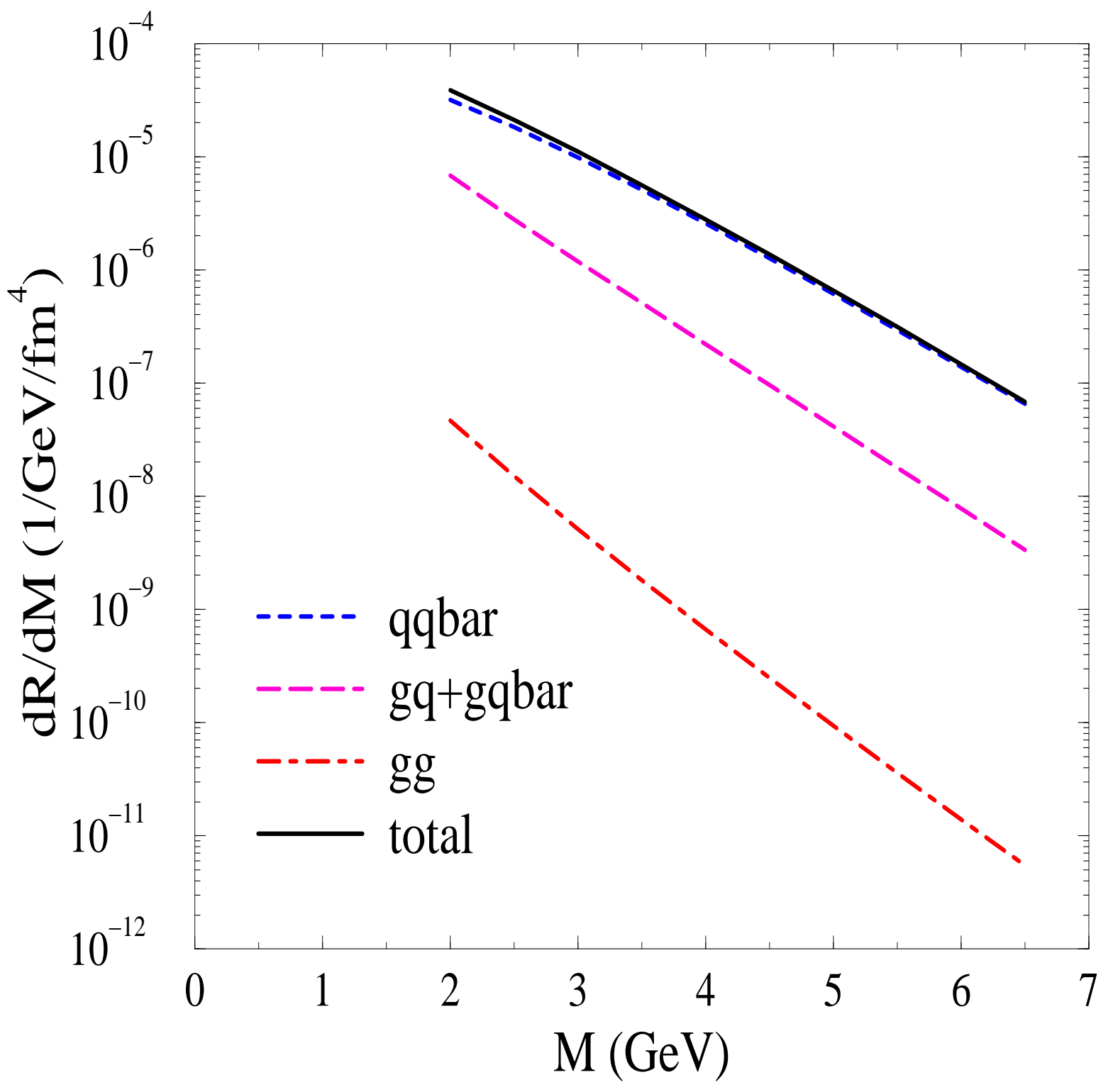

Figure 2: Thermal dilepton rates from a quark-gluon plasma in full thermal and chemical equilibrium at a temperature $T=570 \mathrm{MeV}$. 


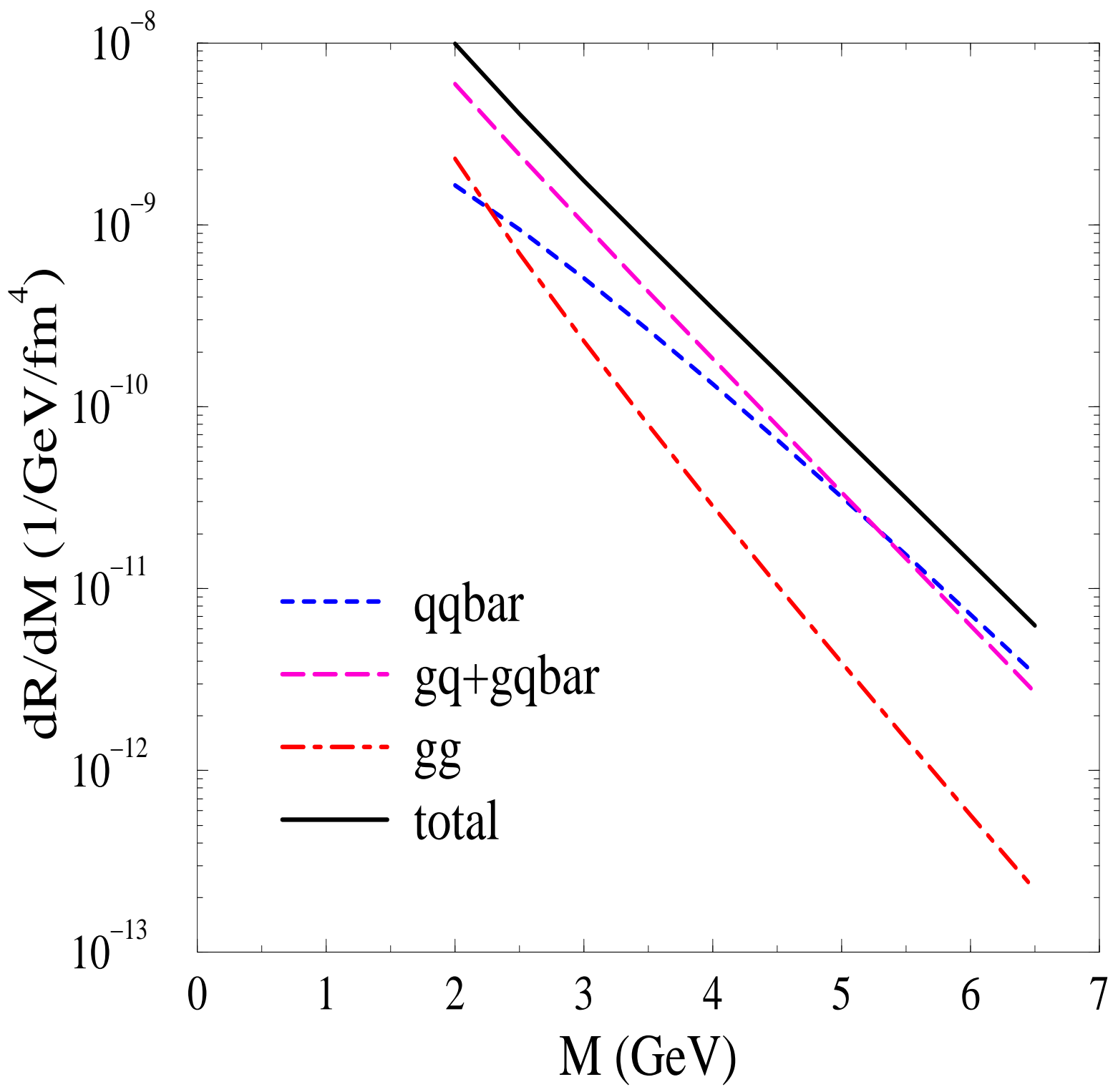

Figure 3: Thermal dilepton rates from a gluon-dominated, under-saturated quarkgluon plasma with $T=570 \mathrm{MeV}, \lambda_{g}=0.060$ and $\lambda_{q}=0.0072$. 


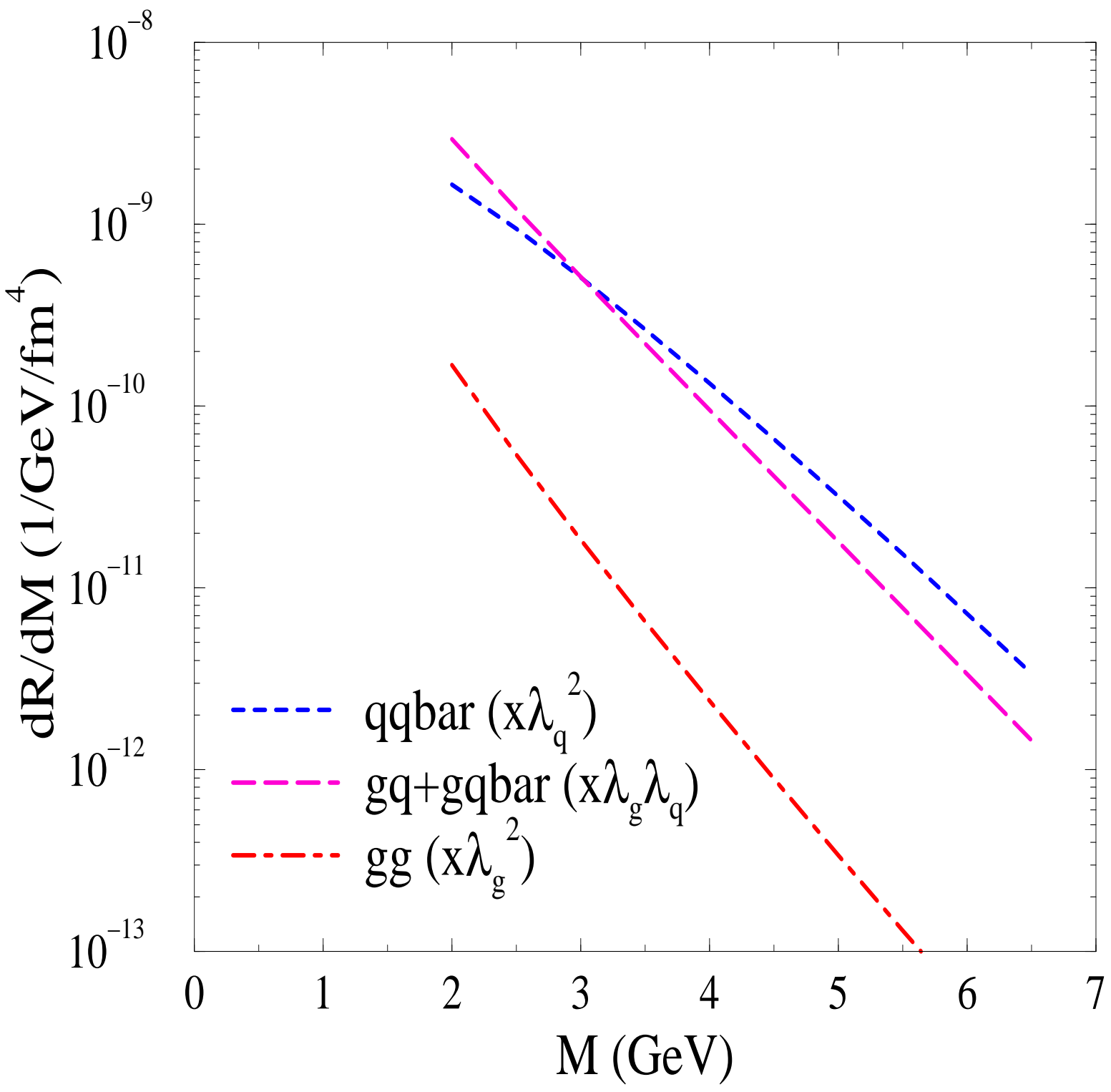

Figure 4: Thermal dilepton rates from a quark-gluon plasma in full thermal and chemical equilibrium at $T=570 \mathrm{MeV}$ after being multiplied by the fugacity factors $\lambda_{g}=0.060$ and $\lambda_{q}=0.0072$. 


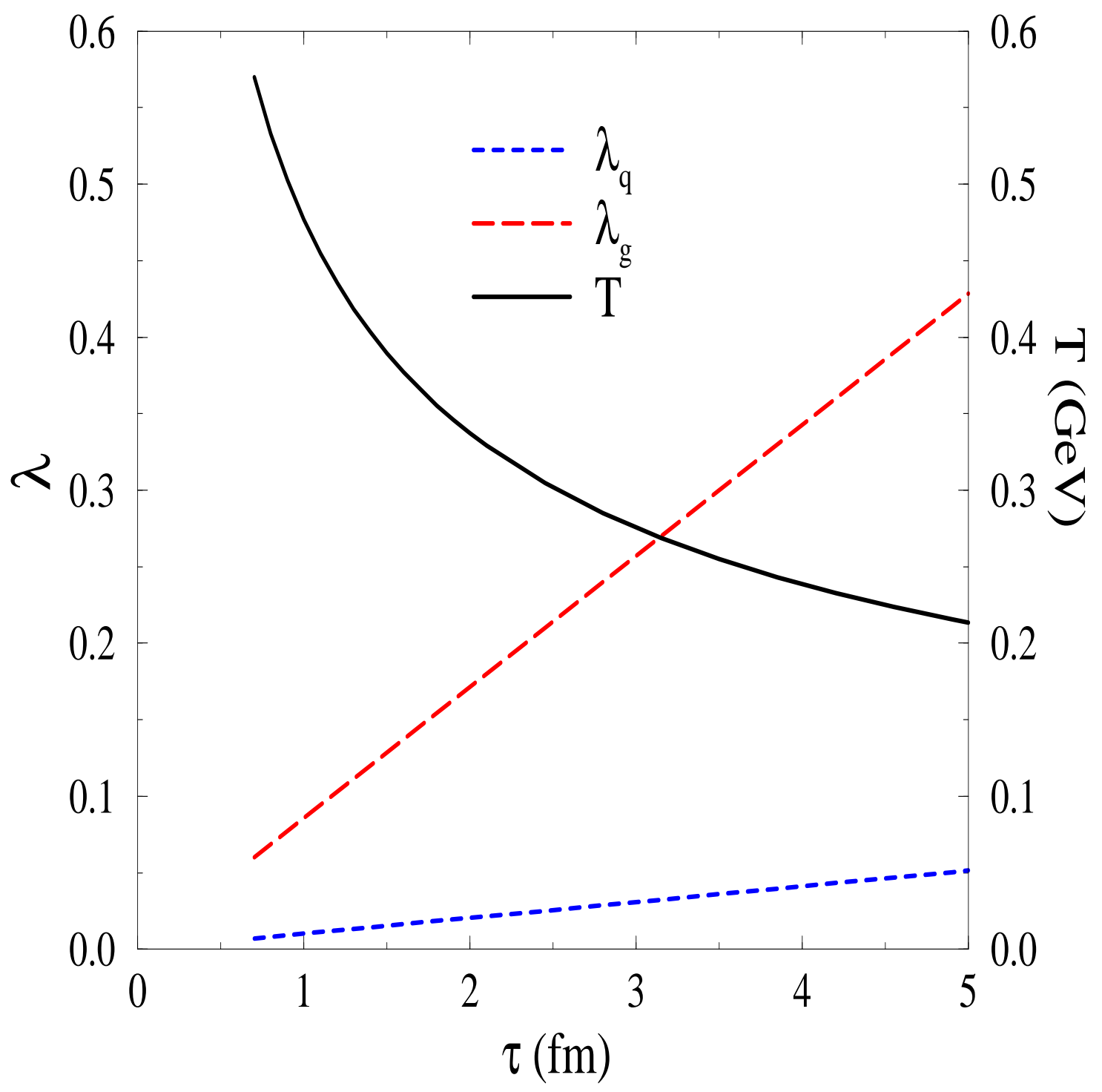

Figure 5: Time evolution of parton fugacities and temperature. 


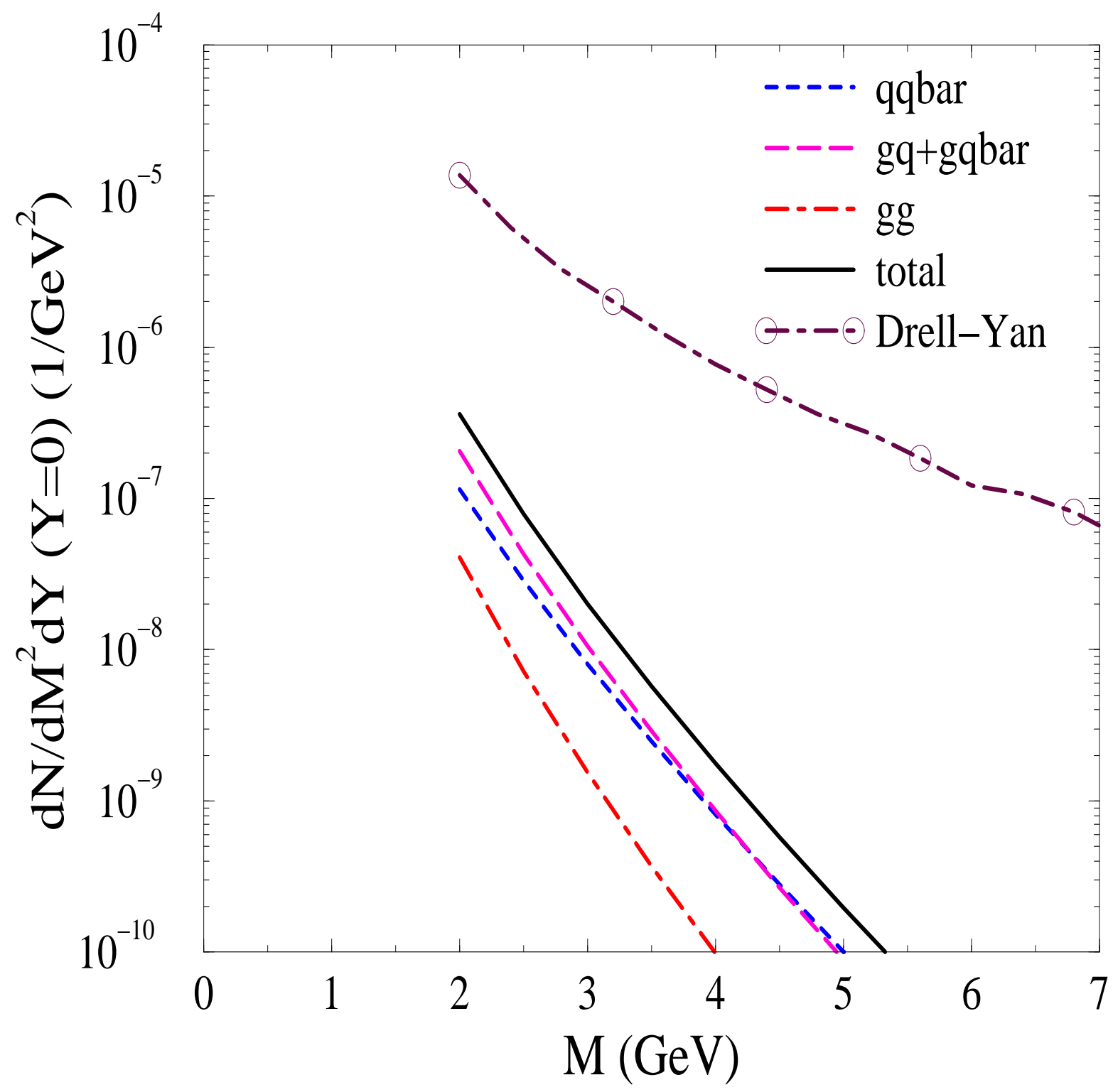

Figure 6: Thermal dilepton yields for central $\mathrm{Au}+\mathrm{Au}$ collisions at $\mathrm{RHIC}$ with initial conditions of $T_{0}=570 \mathrm{MeV}, \lambda_{g, 0}=0.060, \lambda_{q, 0}=0.0072$ and the time evolution according to Eqs.(28) and (30). 


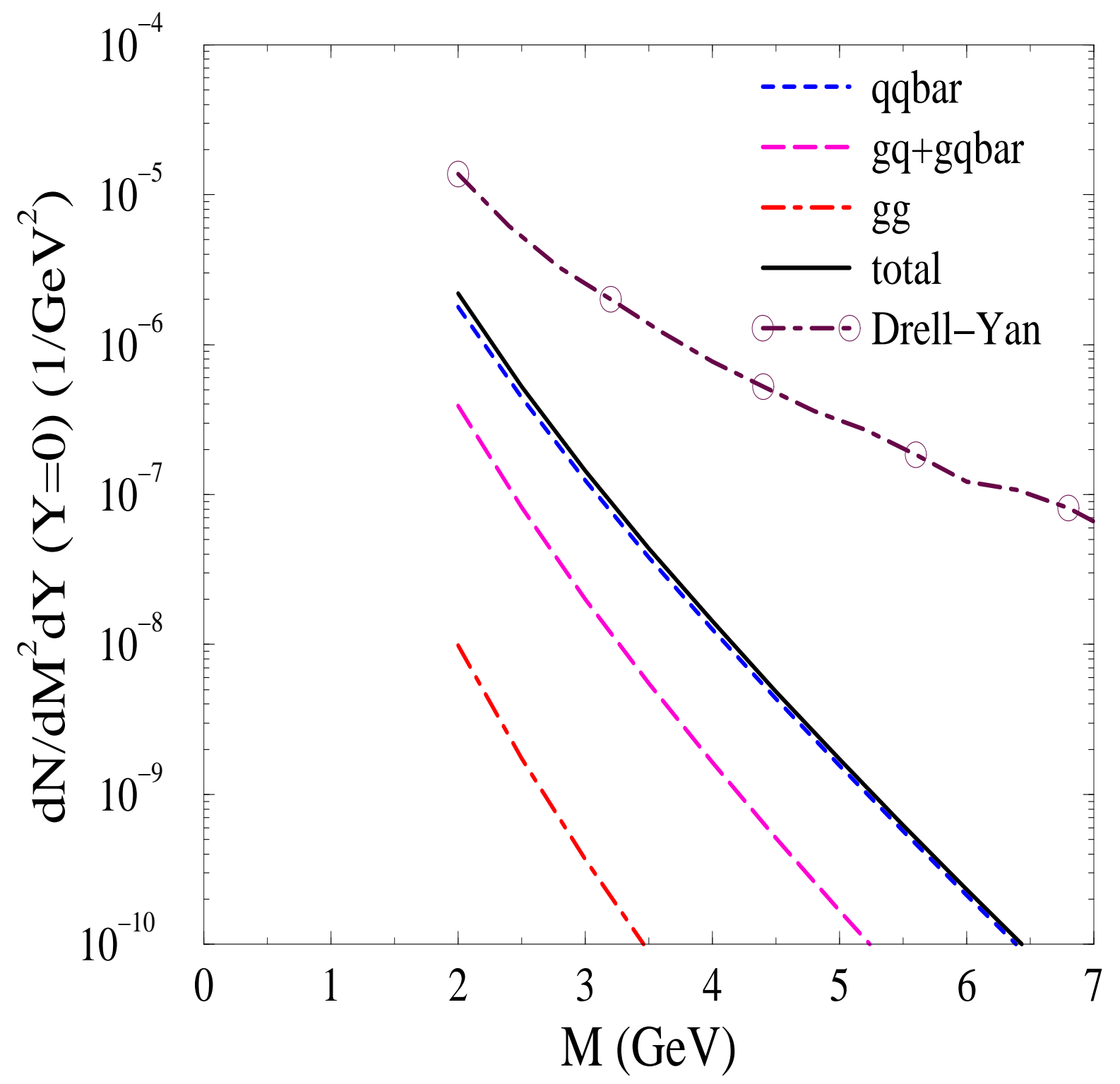

Figure 7: Upper limit of thermal dilepton yields for central $\mathrm{Au}+\mathrm{Au}$ collisions at RHIC with initial conditions of $T_{0}=570 \mathrm{MeV}$ and $\lambda_{g, 0}=\lambda_{q, 0}=0.028$. 


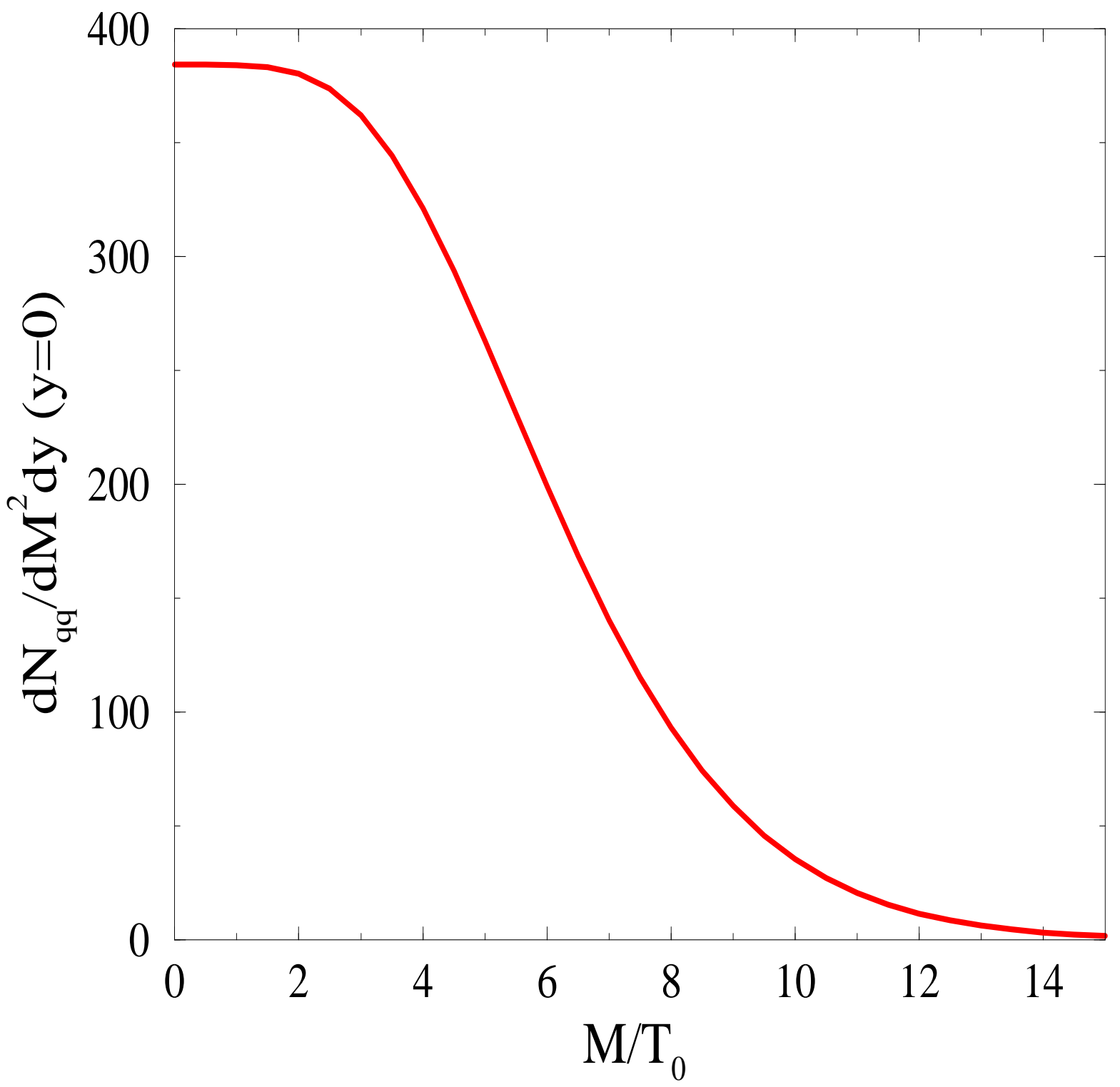

Figure 8: Dependence of the thermal dilepton yield from the LO $q \bar{q}$ annihilation on the initial temperature $T_{0}$ with fixed rapidity density of the initial transverse energy. See Eq.(38) for the unit of the ordinate. 


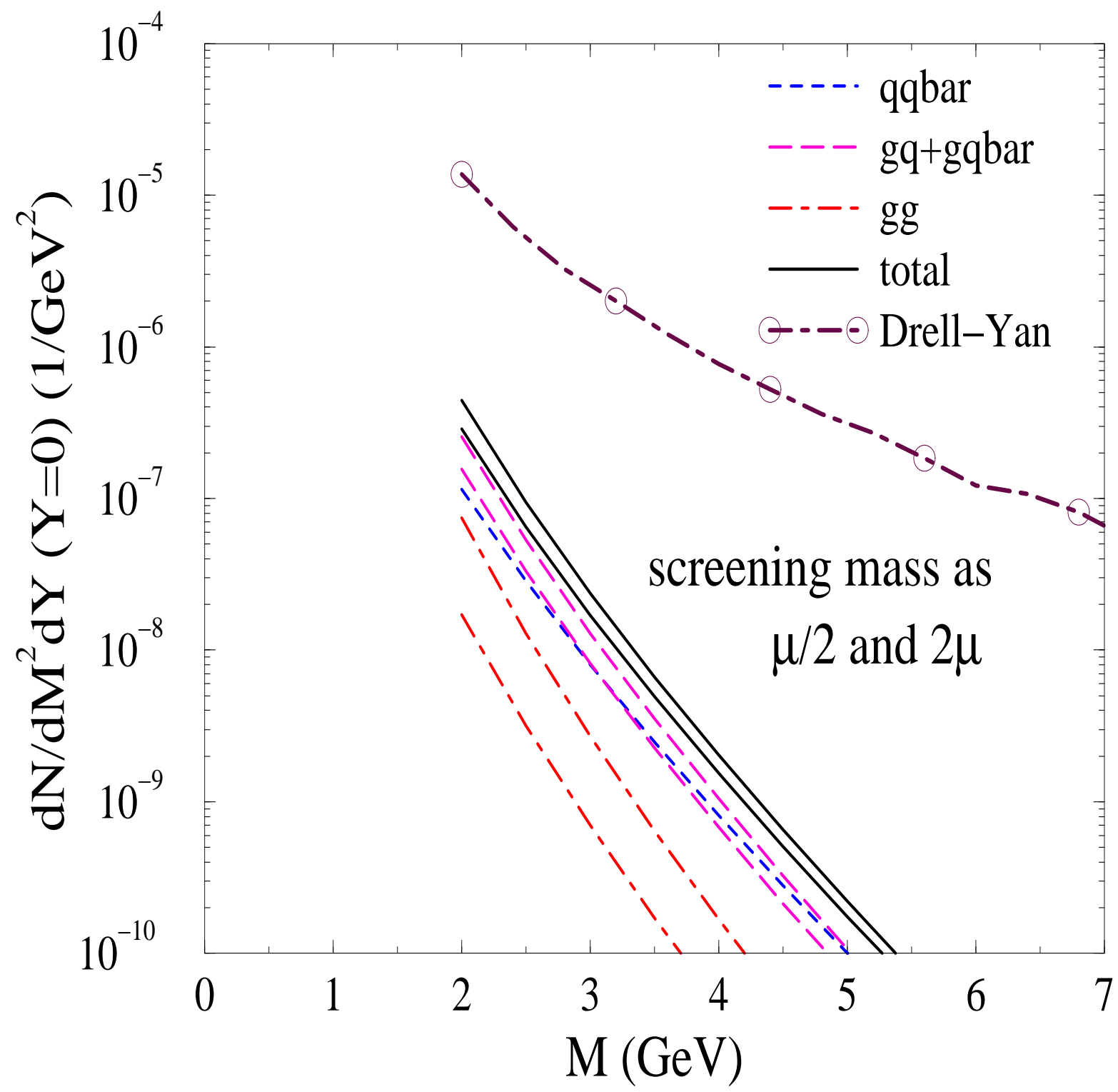

Figure 9: Screening mass dependence of thermal dilepton yields for central $\mathrm{Au}+\mathrm{Au}$ collisions at RHIC with initial conditions of $T_{0}=570 \mathrm{MeV}, \lambda_{g, 0}=0.060$ and $\lambda_{q, 0}=$ 0.0072. Upper and lower curves correspond to results using half and twice the thermal quark mass as the screening mass, respectively. 